\title{
Improving pregnancy outcomes in tertiary care institution in India through obstetric intensive care unit: three-year analysis
}

\author{
Ashish V. Gokhale, Shonali Agarwal*, Dimpi Modi
}

Department of Obstetrics and Gynecology, S. S. G. Hospital, Vadodara, Gujarat, India

Received: 18 February 2020

Accepted: 20 March 2020

\section{*Correspondence:}

Dr. Shonali Agarwal,

E-mail: shonaliagarwal75@yahoo.co.in

Copyright: (C) the author(s), publisher and licensee Medip Academy. This is an open-access article distributed under the terms of the Creative Commons Attribution Non-Commercial License, which permits unrestricted non-commercial use, distribution, and reproduction in any medium, provided the original work is properly cited.

\begin{abstract}
Background: To analyse the requirement of high dependency unit (HDU)/intensive care unit (ICU) in an obstetric population in terms of utilization rate, indications for admission, interventions required and gestational outcome.

Methods: Retrospective observational study was carried out from April 2015 to September 2018 at department of obstetrics and gynecology SSG Hospital, Vadodara India. Data related to indications for ICU admission, interventions required, length of stay and outcomes were collected, and results were analyzed.

Results: Obstetric ICU utilization rate was 130 per 1000 deliveries. The major obstetric indications for admission were hypertensive disorders of pregnancy $(42 \%)$ followed by anemia $(25 \%)$ and post-partum hemorrhage (3\%). In the rest of the patient's majority $(50 \%)$ had cardiovascular problems. Maternal mortality among these critically ill women was $3.76 \%$. The death rate in the present study was high among patients admitted for non-obstetric (74.54\%) as opposed to obstetric indications $(25.46 \%)$.

Conclusions: Establishment of well managed high dependency and intensive care unit in health care facilities dealing with high volumes of high-risk maternity cases reduces the maternal mortality significantly and results in improved maternal outcomes.
\end{abstract}

Keywords: High dependency unit, Intensive care unit, Obstetric critical care, Pregnancy complications

\section{INTRODUCTION}

Confidential enquiry into maternal deaths have revealed that there are still a significant number of maternal deaths associated with suboptimal care.

"There is a story behind every maternal death and lifethreatening complication" and understanding the lessons to be learnt can help to avoid such outcomes. ${ }^{1}$ Any pregnant woman can develop life threatening complications with little or no advance warning. Though pregnancy and labor are considered a physiological process the potential for catastrophic complications is constant and may develop in a matter of minutes. The diagnoses precipitating admission to critical care are predictable and include massive haemorrhage $(>2,500 \mathrm{ml}$ loss), eclampsia, sepsis, thromboembolism, acute organ dysfunction (renal, hepatic, cardiac, respiratory, neurological) and anaesthesia-related morbidities such as aspiration, anaphylaxis and muscle relaxant-related problems.

These women with obstetric complications need access to quality maternal health services that can detect and manage life threatening obstetric complications. They need round the clock personalized care by skilled providers, essentially led by obstetricians or emergency obstetric care (EmOC) trained providers. For those women who have progressed to an adverse clinical condition where there is multi-organ involvement/failure, the care has to be provided in an intensive care unit setting led by intensivist/super-specialists. 
The proportion of obstetric patients requiring admission to intensive care units (ICUs) varies among published series at between 1-9 per 1000 deliveries, with obstetric complications accounting for the majority of admission diagnoses. The availability of a high dependency unit (HDU) within an obstetric setting has a number of potential advantages, including the concurrent availability of expert obstetric care and critical care management. It has been suggested that the presence of 'on-site' critical care facilities would reduce the need to transfer patients to a general medical/surgical ICU. ${ }^{2}$

It has been observed that majority of maternal deaths recorded at the institution have multiple morbidities including obstetric disorders and medical disorders complicating pregnancy. These mothers require specialized intensive critical care and some require ventilatory support.

The presence of a well-developed critical care unit (HDU/ICU) with appropriate infrastructure, equipment's and trained staff is the key for providing necessary and timely intensive management to these critical mothers.

In Western India the incidence of mothers requiring intensive care unit is around 5.48 per 1000 deliveries. ${ }^{3}$ In central rural India in this study retrospectively analyzed the demographic parameters, reason for admission and outcome of obstetric cases requiring admissions in HDU/ICU with an aim to review HDU/ICU setup in terms of HDU utilization rate, indications for admission, interventions needed and maternal outcome..

\section{METHODS}

A hospital based, cross sectional, observational study was conducted in obstetrics and gynaecology department S. S. G. Hospital, Vadodara, Gujarat, India, tertiary care referral centre serving underprivileged rural masses.

\section{Inclusion criteria}

- All antenatal and postpartum patient (42 days of delivery)

\section{Obstetric complication}

- Severe anemia

- Accidental hemorrhaged (abruption, placenta previa)

- Post-partum hemorrhage

- Adherent and other placental morbidity

- Obstetric hysterectomy

- Severe preeclampsia/eclampsia/HELLP syndrome

- Uterine perforation and rupture

- Sepsis

- Thrombophilia

- Multiple gestation with complication

- V. mole

- Ruptured ectopic
- Postoperative patient requiring hemodynamic monitoring

\section{Pregnancy with medical complications}

- Pregnancy with gestational diabetes

- Pregnancy with diabetic ketoacidosis

- Pregnancy with cardiac disease

- Pregnancy with jaundice

- Pregnancy with thyrotoxicosis/ thyroid storm

- Pregnancy with pheochromocytoma

- Pregnancy with other endocrinal crisis like Addison's disease etc.

- Postoperative ARF and other renal problems

- Leukemia and other hemolytic disorders

- Pregnancy with dengue/ complications of malaria

- Pregnancy with asthma and other respiratory problems

- Pregnancy with OHSS (ovarian hyperstimulation syndrome)

- Pulmonary edema due to perioperative fluid overload, CCF, complication of severe pre-eclampsia or tocolytic therapy with beta agonists, etc.

- Pregnancy with DIC

- Pregnancy with cancer.

\section{Exclusion criteria}

- Postpartum patient after 42 days of delivery

- Pregnancy with appendectomy or any other surgical emergency

- Pregnancy with trauma

- Pregnancy with poisoning

- Burns during pregnancy.

The state has a maternal mortality ratio of 112 per one lakh live births (SRS 2011-13) and the health system is striving hard to provide quality maternity care and reduce maternal mortality further. S. S. G. Hospital, Vadodara, Gujarat, India is a centre of excellence with a dedicated team of health care providers and hence most of the complicated maternal cases from the surrounding district's and even adjacent states of Rajasthan and Madhya Pradesh are referred to this center.

The referred cases at institution has further increased due to improved infrastructure in the form of newly constructed maternal and child health wing and availability of ICU facilities.

Keeping the goal of providing a continuum of care with quality and with its endeavour to provide integrated, comprehensive reproductive, maternal, new born, child and adolescent health in the region and showcase a model in the country, Government of India under the National Rural Health Mission and Government of Gujarat which has become functional from April 2015. The department of obstetrics and gynecology believes in providing quality services with a human touch to all the rural and 
urban women visiting the outdoor and indoor sections. The department has facilities for antenatal, postnatal, gynecological care as well as 6 bedded intensive care unit.

\section{Study tool}

Demographic information, relevant obstetric history, the reason for admission in HDU and ICU, need for ventilation, type of interventions and outcome of the pregnancy was obtained in a pretested, validated questionnaire.

The data was collected through hospital information system, from April 2015 to September 2018 and information from January 2014-December 2014 was the control. The comparison was done between obstetric outcome prior to setup ICU and post. Intensive care unit (ICU) was defined as specialised care unit dedicated to obstetric patients who have developed multi-organ failure necessitating specialized care by super-specialists like Intensivist/nephrologist/cardiologist/pulmonologist/endoc rinologist, etc. or requiring ventilatory support (mechanical ventilation). The study team taking care of the women comprised of trained nurses, resident doctors, consultant obstetricians, anaesthetist, Intensivist and physician.

\section{Statistical analysis}

Data was entered in a spread sheet (Excel) and analysis was done.

Table 1: Performance of obstetric facility in $\mathrm{MCH}$ wing after setup of ICU, (2015-18).

\begin{tabular}{|lll|}
\hline Sr. & Indicator Number (n) & \\
\hline 1. & $\begin{array}{l}\text { Total number of deliveries in the } \\
\text { institution 2015-2018 }\end{array}$ & 24,970 \\
\hline 2. & Total admissions to obstetric ICU & 3320 \\
\hline 3. & $\begin{array}{l}\text { Average monthly admissions in } \\
\text { ICU }\end{array}$ & 77 \\
\hline 4. & $\begin{array}{l}\text { Average length of stay in days in } \\
\text { ICU }\end{array}$ & 03 \\
\hline 5. & Bed occupancy rates of ICU & $90 \%$ \\
\hline 6. & $\begin{array}{l}\text { No. of patients successfully } \\
\text { discharged (home ward) from ICU }\end{array}$ & 2988 \\
\hline 7. & No. of patients referred & 181 \\
\hline 8. & $\begin{array}{l}\text { No. of patients DAMA, DOR, } \\
\text { LAMA }\end{array}$ & 25 \\
\hline 9. & No. of patients expired & 125 \\
\hline
\end{tabular}

Year wise distribution of indication of admission suggested major group of admission were eclampsia followed by anemia.

As per Figure 2, maximum women were in the age group of 21-25 years followed by age group of 26 to 30 years

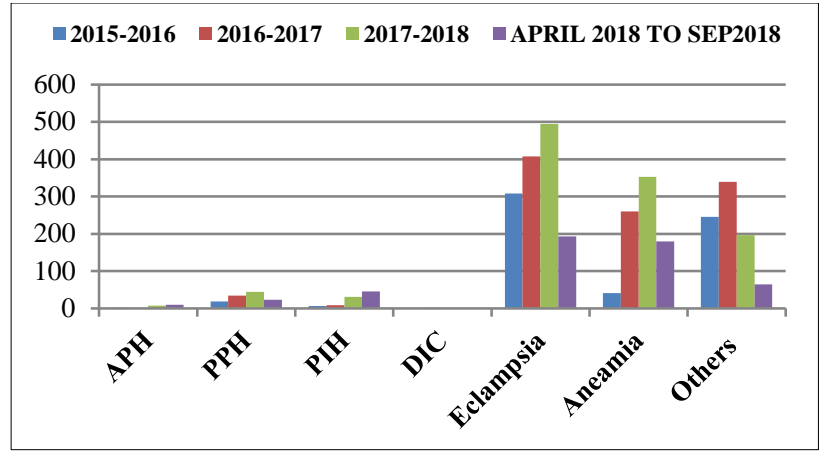

Figure 1: Indications for admission in obstetric ICU.

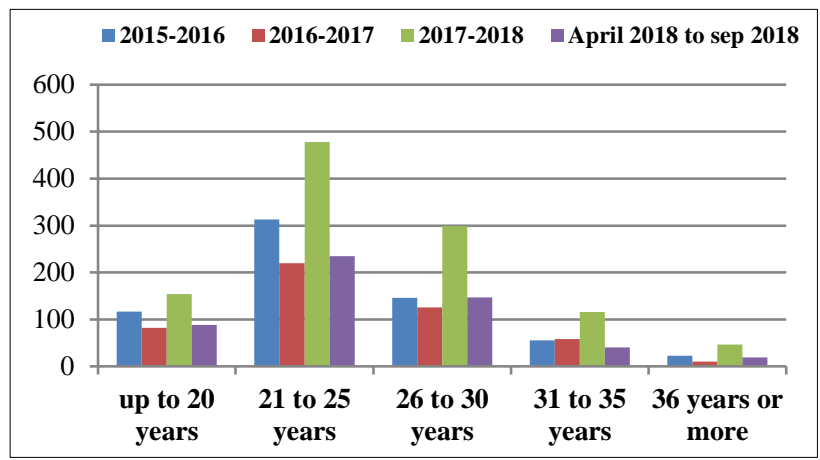

Figure 2: Age wise distribution of patients admitted in obstetric ICU.

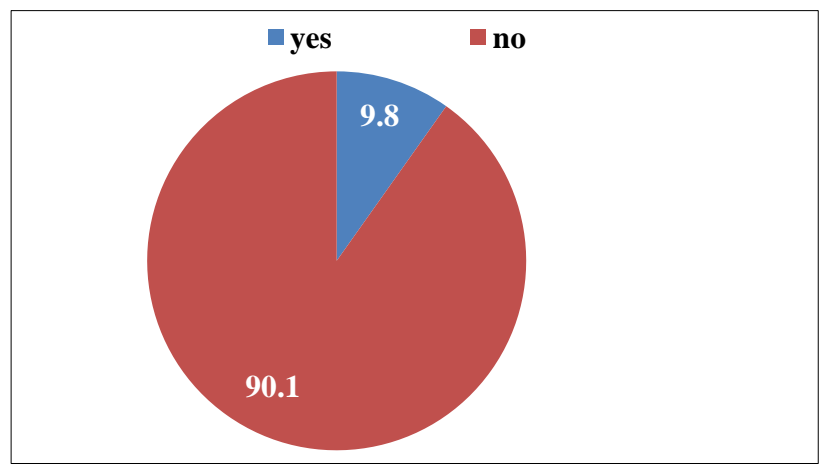

Figure 3: Percentage of women requiring ventilatory support.

As per Figure 3, 90.1\% patients required ventilator support.

As per Figure 4, year wise distribution of various Interventions carried out were 1. blood transfusion 2 . antihypertensive drug administration 3. anticonvulsant (inj. magnesium sulphate) 4 . ventilatory support 5 . obstetric hysterectomy.

As per Figure 5, most women; (90\%) were successfully discharged home-ward and 125 expired (3\%).

As per Figure 6, the average length of ICU stay was 3-3.5 days. 


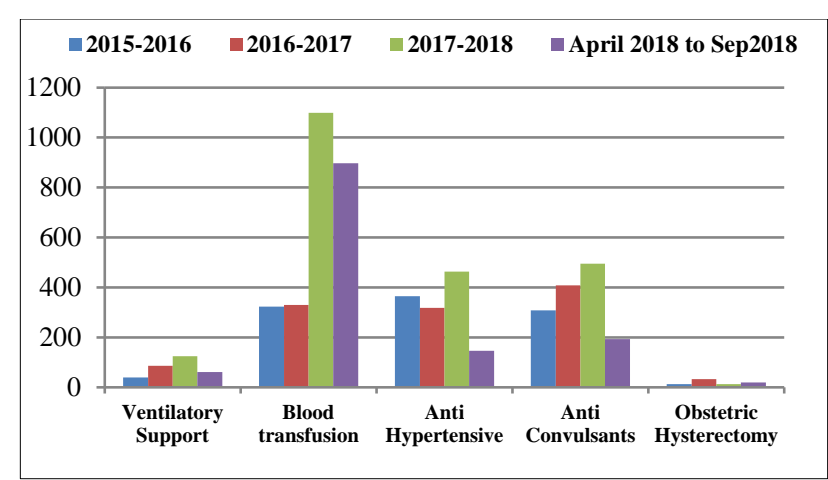

Figure 4: Interventions carried out on women admitted in obstetric ICU.

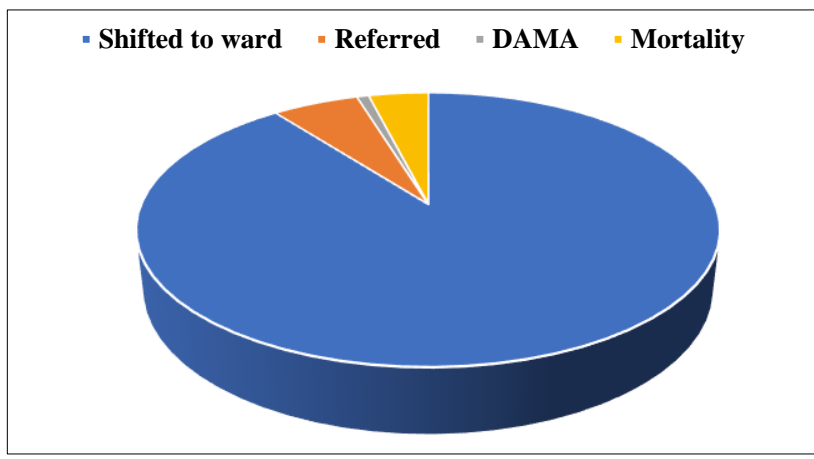

Figure 5: Outcome of women admitted in obstetric ICU.

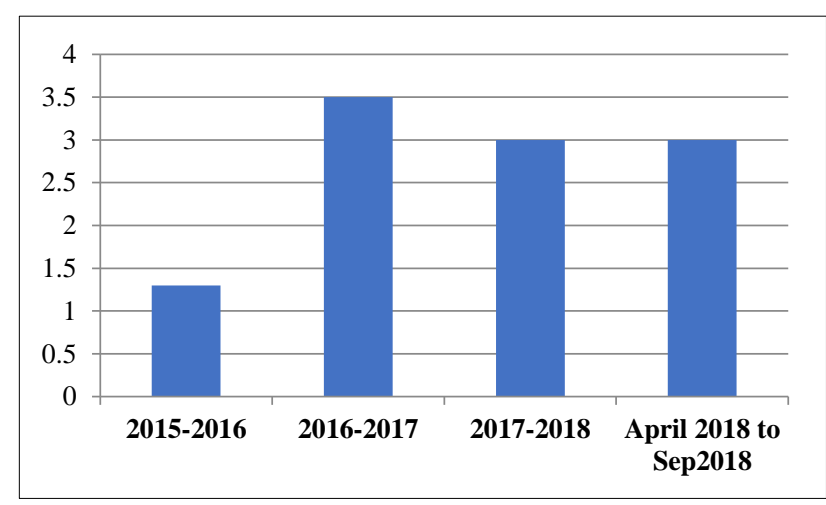

Figure 6: Average length of ICU stay.

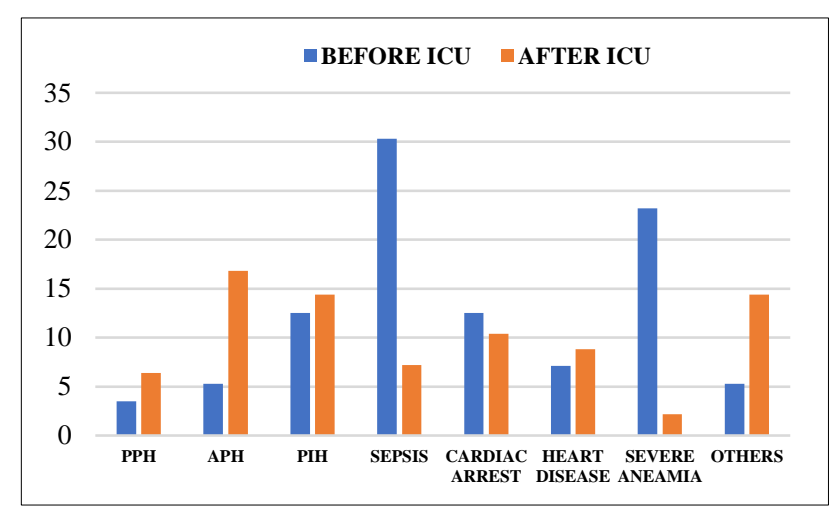

Figure 7: Causes of maternal mortality.
Figure 7, enumerates various causes of maternal mortality before and after establishment of obstetric ICU. The contribution of sepsis and severe anemia to mortality has drastically fallen suggestion efficiency of obstetric ICU setup.

\section{RESULTS}

During the period of study, there were 3320 admission.

During the study period, there were Approximately 30000 obstetric admissions, 24,970 mothers delivered and $3320(13 \%)$ required care in intensive unit.

The average monthly admissions were 77 with an average length of stay of 3 days and average bed occupancy was $90 \%$.

Maximum women were in the age group of 21-25 years (Figure 2).

A total $9 \%$ women received ventilatory support (Figure $3)$.

Most women; (90\%) were successfully discharged homeward and 125 expired (3\%) (Figure 5).

Heart disease, sickle cell disease with crisis, jaundice and infections accounted for non-obstetric causes and the average length of ICU stay was 3-3.5 days (Figure 8).

Length of ventilatory support dictated the maternal survival and more than 3 days of advanced respiratory support carried a significant risk of mortality.

Significant improvement in outcome of patients was seen after the establishment of functioning as evident from the reduction in maternal mortality.

\section{DISCUSSION}

Intensive care is appropriate for patients who require or are likely to require advanced respiratory support alone, for those requiring support for more than one organ system or for patients with chronic impairment of one or more systems sufficient to restrict daily activities (comorbidity) and who require support for an acute reversible failure of another organ system.

High acuity pregnancy related illnesses necessitating level of cardiovascular and respiratory monitoring exceeding 'normal' practice in delivery units have been recorded in significant number of women with a range of diagnoses; from small post-partum hemorrhages $(>1,500$ $\mathrm{ml}$ ), to pre-eclampsia, uterine rupture and HELLP syndrome.

Among patients with obstetric hemorrhage, majority had postpartum as compared to antepartum hemorrhage $(3 \%$ versus $0.6 \%$ ). 


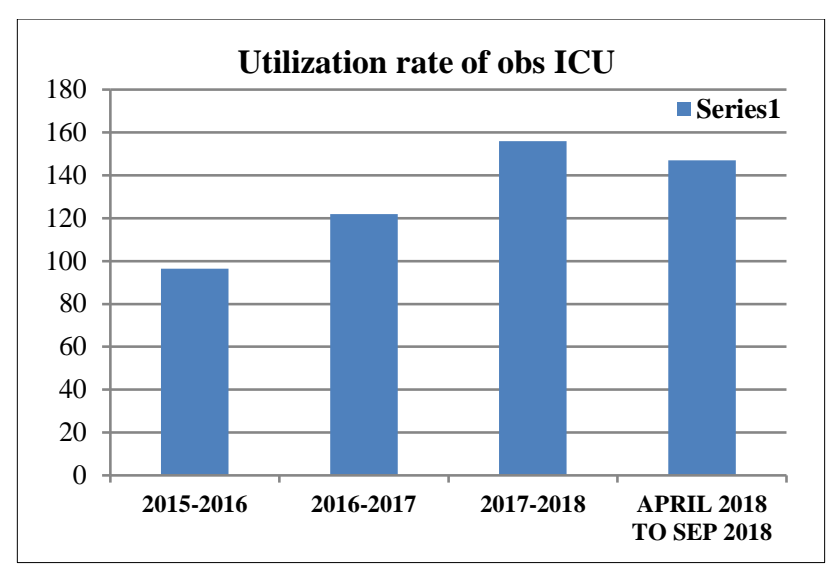

Figure 8: Performance of obstetric ICU.

However, Dattaray et al, found that anemia and sepsis accounted for the majority (35.08\%) of admissions, following by severe hypertensive disorders of pregnancy (42\%). Common causes of sepsis were massive obstetric hemorrhage, chorio-amnionitis, urinary tract infections, puerperal infections and sequelae of operative interventions including criminal abortions.

Home deliveries in rural India or at health centres with inadequate infrastructure, unsupervised deliveries, dogmatic traditional approaches during birth, failure to use uterotonics and underlying anemia continue for PPH amounting to almost $28 \%$ of maternal mortality in India.

An audit of severe maternal morbidity in Dublin, with 13 systems and management-based definitions, showed a rate of 320/100,000 for massive obstetric hemorrhage, as defined by the requirement of greater than or equal to five units of blood transfusion, occurred in 125/100,000 deliveries in one large multicenter study. It has been observed that anemia and poor nutrition contribute to the risk of hemodynamic compromise arising from blood loss during delivery and this increases the maternal morbidity.

In women with hypertensive disorders of pregnancy requiring HDU care, lack of timely diagnosis and inappropriate management coupled with ignorance of life-threatening complications like HELLP and eclampsia were responsible for poorer outcome.

Among non-obstetric complications various medical disorders like rheumatic heart disease, infective hepatitis, sickle cell disease with crisis, acute respiratory distress syndrome, dengue, renal dysfunction, H1N1 etc. necessitated HDU admission. Similar observations were reported by other researchers, though sickle cell disease with crisis is observed to be more common in this particular region of central, rural India. ${ }^{2.4}$ Acute, intensive maternal disease related morbidities were significantly increased in the obstetric cases with prior medical illness, and these included cardiac failure, respiratory failure and prolonged hospital stay and increased recovery time. A total of $12 \%$ of patients required advanced respiratory support in the form of mechanical ventilation. Another study conducted in China reports it to be as high as $40 \% .^{1}$ The most frequent indications were acute respiratory failure $(21 \%)$ and hemodynamic failure $(41 \%)$.

The mortality rate of $3 \%$ for obstetric ICU admissions the present study is below the developed world median of $3.4 \%$ (range $0-18.4 \%$ ) and the developing world median of $14.0 \%$ (range $0-40 \%$ ) which shows a dedicated team effort and advocates the establishment of wellfunctioning obstetric critical care units in developing countries like India. ${ }^{5}$ Other studies report maternal death rate in the ICU varying from $3.4-21 \% .^{6,7}$

Inadequate knowledge about the illness, infrequent admission of obstetric patients and non-availability of critical care setup results in high mortality and morbidity. The death rate in the present study was high among patients admitted for non-obstetric $(74.54 \%)$ as opposed to obstetric indications $(25.46 \%)$ thus implying the need for concentrating not only on good antenatal care but also prompt diagnosis of medical conditions and appropriate management in multidisciplinary setups wherein more attention needs to be focused on life threatening obstetric conditions by skilled obstetricians. Dattaray et al, also found that patients with medical disorders had more mortality in comparison to obstetric group $(5 / 18$ versus $2 / 39 ; \mathrm{p} \leq 0.05) .{ }^{8,9}$

High mortality rate as quoted in various studies was found in this study due to late referral, $2^{\text {nd }}$ or $3^{\text {rd }}$ from the peripheral centers, lack of awareness about the disease severity by the community, delay in transportation, and delay in initiation of the treatment. ${ }^{6}$

Another study found high maternal age, absence of prenatal care, transfer to ICU > 24 hours after onset of acute problem and severity of illness at the time of admission as assessed by APACHEII score to be the factors responsible for maternal mortality. ${ }^{10}$ Early detection and timely appropriate intervention might avoid or minimize the effects of such complications.

Proved treatment strategies for obstetric hemorrhage and sepsis along with timely management of critically ill patients in the high-dependency unit may help decrease maternal mortality and morbidity as a high-dependency care unit fulfils the needs of at least half of the obstetric population who require higher acuity care and simultaneously save considerable expenditure.

Emphasis on early detection of maternal problems and prompt referral to tertiary centers could minimize the prevalence of multiple organ failure and mortality in these women. Study findings highlight the need for establishing a high dependency unit in all such centres in order to avoid unnecessary admission to the intensive care unit and to ensure proper management. Again, since puerperal morbidity has now become a focal parameter for quality of maternal care, a continued psychosocial 
follow-up of these patients should ideally be implemented to ensure better quality of life. Constant vigilance, judicious judgment along with provision of adequate resources are still the major stepping stones on the road to maternal safety. ${ }^{9}$

The focus of interest should shift to intermediate care due to inadequate supply of intensive care facilities as a tool of bridging the gap between the level of care and support available in general ward and intensive care unit. Some hospitals have developed intermediate care in the form of HDU, however the information regarding the use of such units delivering quality care is very limited.

Staff caring for patients in acute hospital settings should have competences in monitoring, measurement, interpretation and prompt response to the acutely ill patient appropriate to the level of care they are providing. Education and training should be provided to ensure staff have these competencies and they should be assessed to ensure they can demonstrate them. ${ }^{11}$

Developing team work, assertiveness and interprofessional working relationships, monitoring outcomes at all levels and critical incident analysis is imperative for ongoing improved maternal outcomes. Implementing these competences will require an effective leadership with system-wide approach and rigorous change management. The principle in managing the acutely ill pregnant woman is optimal management of the condition, with a multidisciplinary team. The present study reinforces and strongly advocates establishment of dedicated obstetric HDU/ICU especially in developing countries like India, to deliver a continuum of skilled obstetric care which promptly and skillfully addresses acute complications, chronic disorders getting amplified during pregnancy and catastrophic events through pregnancy, childbirth and post-partum so as to reduce maternal morbidity and mortality significantly.

\section{CONCLUSION}

Anemia and pregnancy-related hypertension with its complications are the two common indications for ICU admissions in this study. Other than these two common obstetric indications, medical disorders like heart disease, respiratory diseases and their complications, infections are prevalently seen in pregnant women with acute morbidity requiring ICU admissions.

A dedicated obstetric HDU/ICU will go a long way in reducing maternal mortality as the response time from onset of acute severe illness to provision of high-quality critical care will be substantially reduced.

A multidisciplinary team approach with trained staff for timely quality management is the crux for continuum of skilled obstetric and critical care in pregnant women.
Hence, the need for a dedicated obstetric ICU/HDU or critical care unit should be considered in every large public hospital and tertiary health care facilities catering to high risk pregnant women.

\section{ACKNOWLEDGMENTS}

Authors acknowledge sincerely the support of faculty and postgraduates of obstetrics and gynecology, medicine, anesthesiology and preventive and social medicine department, S. S. G. Hospital Vadodara, Gujarat, India.

Study also acknowledge the Ministry of Health and Family welfare for providing the maternal and child health wing a dedicated high dependency unit and intensive care unit.

Funding: No funding sources Conflict of interest: None declared

Ethical approval: The study was approved by the Institutional Ethics Committee

\section{REFERENCES}

1. Leung NY, Lau AC, Chan KK, Yan WW. Clinical characteristics and outcomes of obstetric patients admitted to the Intensive Care Unit: a 10-year retrospective review. Hong Kong Med J. 2010;16(1):18-25.

2. Ryan M, Hamilton V, Bowen M, McKenna P. The role of a high-dependency unit in a regional obstetric hospital. Anaesth. 2000;55(12):1155-8.

3. Karnad DR, Lapsia V, Krishnan A, Salvi VS. Prognostic factors in obstetric patients admitted to an Indian intensive care unit. Critical Care Med. 2004;32(6):1294-9.

4. Zwart JJ, Dupuis JR, Richters A, Öry F, van Roosmalen J. Obstetric intensive care unit admission: a 2-year nationwide population-based cohort study. Intens Care Med. 2010;36(2):256-63.

5. Pollock W, Rose L, Dennis CL. Pregnant and postpartum admissions to the intensive care unit: a systematic review. Intens Care Med. 2010;36(9):1465-74.

6. Collop NA, Sahn SA. Critical illness in pregnancy: An analysis of 20 patients admitted to a medical intensive care unit. Chest. 1993;103(5):1548-52.

7. Platteau P, Engelhardt T, Moodley J, Muckart DJJ. Obstetric gynaecological patients in an intensive care unit: a 1 year review. Trop Doctor. 1997:202-6.

8. Ridley SA. Intermediate care possibilities, requirements and solutions. Anaesth. 1998;53(7):654-64.

9. Dattaray C, Mandal D, Shankar U, Bhattacharya P, Mandal S. Obstetric patients requiring highdependency unit admission in a tertiary referral centre. Inte J Critic Ill Inj Sci. 2013;3(1):31.

10. Bhat PB, Navada MH, Rao SV, Nagarathna G. Evaluation of obstetric admissions to intensive care 
unit of a tertiary referral center in coastal India. Indian J Crit Care Med. 2013;17(1):34.

11. Chalkley S. Acute illness essentials Care of the Acutely Ill Adult: An Essential Guide for Nurses Fiona Creed and Christine Spiers ISBN: 9780199564385 Oxford University Press 2010, $£ 27.95$. Br J Card Nurs. 2011:480.
Cite this article as: Gokhale AV, Agarwal S, Modi D. Improving pregnancy outcomes in tertiary care institution in India through obstetric intensive care unit: three-year analysis. Int J Reprod Contracept Obstet Gynecol 2020;9:1965-71. 